\title{
The New Strategy in Head and Neck Cancer-Changes in Diagnosis and Survival
}

\author{
Abrão Rapoport* and Otávio Alberto Curioni \\ Head and Neck Cancer Department of Hospital Heliópolis, São Paulo, Brazil
}

*Corresponding author: Abrão Rapoport, Head and Neck Cancer Department of Hospital Heliópolis, São Paulo, Brazil.

Received Date: February 23, 2021

Published Date: March 16, 2021

\section{Opinion}

The approach for Head and Neck Cancer (HNC) is directly related to the predominance (90\%) of Squamous Cell Carcinoma (SCC), where the transition from pre-cancer status to neoplastic clinical lesion is related to stromal inflammation and immune deflection [1]. This focus explain the clinical evolution of the disease and the prognosis without interference of the therapy, where a serie of HPV mucosal viruses determine an oncogenic high risk [2]. Beside the large number of SCC, is mandatory the recognition of a phenothipics changes in these neoplasias, where a great variance of behavior must be considered [3].

These facts indicate a deeply histopathologic assessment looking for immnunological feactures through in situ hibridization and polimerase chain reaction (PCR). Then, determination of HPVs and p53 are important for the evaluation of SCC cells considering the affection of molecular levels after the virus entrance in the DNA of the patient with (HNC) [4]. Considered the epidemiology and the evolution of HNC, an incontrolled cell division, invasion, apoptosis and proliferation (regional or distant metastasis), are usual. Then, we consider that surgery alone or associated to irradiation are local therapies, and chemotherapy is the target method that can destroy the neoplasia through nanoparticles fixed by surface vesides (ligand cells) into receptores of neoplasis cell [5]. When viruses have affinity for cancer cells receptors, the prognosis is better independent of the therapy [6].

After the initial consideration, we consider that molecular alternations in HNSCC as the heterogenicity lost, genic activation or inactivation, justify an inbalance of the results of therapies and overall survival for malignant disease. The HNSCC must be viewed, nowadays, from the perspective of its systemic and multifactorial character, not simply a regional locus. There must be a paradigm shift from a reactive to a predictive, preventive and personalized approach. In this scenario, recent advances in the power of computing and big data manipulation encourage the use of artificial intelligence (AI) to assist or replace conventional approaches. Today, AI-based medical platforms support diagnostics, treatments and prognosis assessments [7]. However, a data-based approach to health care will require doctors to be able to ask the right questions and adapt to interdisciplinary teams.

As conclusion, a new strategy for HNSCC must be considered after the discovery of molecular alternations associated with clinical status of the malignant disease.

\section{Acknowledgement}

None.

\section{Conflict of Interest}

No conflict of interest

\section{References}

1. Marioni G, Altavilla G, Busatto G, Blandamura S, De Filippis C, et al. (2003) Detection of human papillomavirus in temporal bone inverted papilloma by polymerase chain reaction. Acta Otolaryngol 123(3): 367371.

2. S Sangwan, R Seth (2020) Nanotechnology: A boon in câncer therapy: Review. Intern J of Nanomaterials, Nanotechnology and Nanomedicine 2455-3492.

3. O Marzouk, F Brasch, I Todt, P K C Goon, H Sudhoff (2021) Malignant Transformation of Temporal Bone Schneiderian Papilloma Associated with HPV-6. Case Report in Otolaryngology 1:1-4. 
4. Y Shao, Y Song, S Xu, S Li, H Zhou (2020) Expression profile in carcinoma of squamous cell of oral cavity. Front Oncology 10: 1-8.

5. F Memen Heravi, S Bala (2018) Extracellular vesicles in oral squamous carcinoma carry oncogenic miRNA profile and reprogram monocytes via NF- $\kappa$ B pathway. Oncotarget 9(78): 34838-34854

6. F López, MD Williams, A Cardesa, J L Hunt, P Strojan, et al. (2017) How phenotype guides the management of non-conventional SCC of tho larynx. Eur Arch Otorhinolaryngol 7: 2709-2726.
7. BA Tama, DH Kim, G Kim, SW Kim, S Lee (2020) Clinical and Experimental Otorhinolaryngology. Recent Advances in the Application of Artificial Intelligence in Otorhinolaryngology-Head and Neck Surgery 13: 326339. 